\title{
Low yield of routine stress testing in patients awaiting liver transplantation
}

\author{
Cigdem Akincioglu, MD, FRCPC, FEBNM, a and Saurabh Malhotra, MD, MPH, \\ FASNC $^{\mathrm{b}, \mathrm{c}}$ \\ a Division of Nuclear Medicine, Department of Medical Imaging, University of Western Ontario, \\ London, ON, Canada \\ b Division of Cardiovascular Medicine, Jacobs School of Medicine and Biomedical Sciences, \\ University at Buffalo, Buffalo \\ c Clinical and Translational Research Center, Buffalo, NY
}

Received Aug 7, 2018; accepted Aug 8, 2018

doi:10.1007/s12350-018-1409-2

\section{See related article, pp. 254-265}

Among patients with end stage liver disease (ESLD), liver transplantation (LT) is not only guided by clinical need, but also by suitable candidacy to ensure acceptable survival. LT is a high-risk surgical procedure, and the presence of coronary artery disease (CAD) has been associated with high mortality. As such, preoperative cardiovascular evaluation and cardiovascular testing is considered a clinical standard in most patients awaiting LT. Due to a limited number of donors with an increasing need for LT, there is an epidemiological shift wherein transplant recipients are now 'older', which translates into an increasing prevalence of CAD among them. However, patients with ESLD may have an overall lower burden of traditional CAD-risk factors. These patients often do not have hypertension, and the impaired production of lipids by the liver may reduce serum cholesterol levels as well. ${ }^{1,2}$ In addition, as opposed to renal transplant patients, the presence of diabetes mellitus (DM) in ESLD patients is significantly lower. ${ }^{3}$ Despite differences in cardiovascular risk factors when compared to patients receiving other solid organ transplants, small studies have reported a high prevalence of CAD, and as such, up to a 3rd of those awaiting LT may have some evidence of CAD. ${ }^{1}$ In addition, those

Reprint requests: Saurabh Malhotra, MD, MPH, FASNC, Clinical and Translational Research Center, 875 Ellicott Street, Suite 7030, Buffalo, NY 14203; drsmalhotra@hotmail.com

J Nucl Cardiol 2020;27:266-8.

$1071-3581 / \$ 34.00$

Copyright (C) 2018 American Society of Nuclear Cardiology. with nonalcoholic steatohepatitis (NASH) have a higher prevalence of CAD, when compared to those with alcoholic liver disease. ${ }^{4}$ Although the American Association for the Study on Liver Diseases (AASLD) and the American Society of Transplantation collectively recommend routine cardiovascular risk stratification in all patients prior to $\mathrm{LT}^{2}$ the diagnostic and prognostic yields of such an evaluation are not well established.

In this issue of the Journal, Duvall et al summarized their 10-year experience of cardiovascular risk stratification using SPECT MPI, in patients awaiting LT. ${ }^{5}$ The authors evaluated clinical and SPECT MPI data from 34,551 patients who underwent stress MPI between 2004 and 2014. Of these, 2500 patients were evaluated pre-LT. When compared to the rest of the population undergoing SPECT MPI, pre-LT patients were younger, had a lower burden of traditional of CAD-risk factors and a lower prevalence of prior CAD. However, due to their overall poor health associated with ESLD, over $90 \%$ of the pre-LT patients underwent pharmacologic stress testing compared to $49 \%$ of patients who were evaluated for other clinical indications. The authors described several key findings that are highlighted as follows:

1. Among pre-LT patients, an abnormal SPECT MPI was only noted in $7.8 \%$, and this was much lower (5.9\%) among those without prior CAD.

2. A greater burden of CAD-risk factors $(\geq 3$ vs. $<2)$ was associated with greater odds of an abnormal SPECT MPI on univariable regression analysis; however, increasing age was the only independent predictor of an abnormal MPI.

3. Among pre-LT patients without known CAD, but with an abnormal SPECT MPI $(n=142), 45 \%$ $(n=64)$ underwent invasive coronary angiography 
(ICA) which showed obstructive CAD to be present in only 25 (39\% of 64 patients).

4. A minority of the pre-LT patients $(n=271)$ underwent repeat testing, of which $92 \%$ were normal. The mean interval between repeat tests was 27 months.

5. Of all the stress MPI studies performed in pre-LT patients, approximately $84 \%$ were stress-first MPI (80\% were normal) with the need for repeat rest imaging in only $4 \%$ of those undergoing stress-first MPI.

The authors should be commended for providing a detailed descriptive analysis of the use of SPECT MPI in the largest cohort till date of pre-LT patients. Analysis of clinical and imaging data from this large cohort of preLT patients suggests a low yield of routine stress MPI for pre-LT risk stratification, with a limited role of repeat testing. Preselection of patients based on age and multiple CAD-risk factors is likely to provide both clinical and cost effectiveness, so is the use of stress-first imaging. However, this report by Duvall et al does have some limitations that restrict the generalizability of their results. In their study, an abnormal SPECT MPI is suboptimally defined as the presence of either of myocardial infarction or ischemia. This definition clouds the determination of the rationale of repeat testing among patients in this cohort. Furthermore, a low left ventricular ejection fraction (LVEF) and abnormal regional wall motion were excluded from the criteria for an abnormal SPECT MPI. This is particularly important, as a reduced LVEF is a predictor of cardiac mortality, which is the rationale for routine testing and repeat testing in pre-LT patients. This exclusion of low LVEF from their definition of an abnormal SPECT MPI could be the reason for a high false-positive rate ('abnormal' SPECT MPI and nonobstructive CAD) among patients who underwent ICA. In this study, among patients with no known CAD and an abnormal MPI, 64 patients were referred for ICA, while only 25 (39\%) had evidence of obstructive CAD. It is possible that this could be due to artifacts secondary to splenomegaly and ascites in these patients, and referral based on incorporation of LVEF and regional wall motion assessment could have improved the specificity. The authors reported performing stress-first SPECT MPI in the majority of their patients and this is truly remarkable. However, they do not elaborate on their criteria for selection of patients for stress-first imaging. It is conceivable that in a laboratory with access to high-resolution cadmium-zinc-telluride (CZT) SPECT cameras, stress-first imaging can be employed in almost everyone without a prior history of CAD. However, this may not be true for imaging with standard Anger cameras, where a careful preselection of patients would be required to ensure that rest imaging could be avoided in the majority. Overall, this study by Duvall et al compliments the results of prior smaller studies that have suggested a relative low risk of CAD in ESLD patients. Still questions remain as to whom to test, how to test, and how often.

Currently, there is no consensus regarding cardiovascular testing in pre-LT patients, which is likely due to the heterogeneity of the published data and how CAD is defined. Contrary to the perfusion data presented by Duvall et al, ${ }^{5}$ small studies of anatomical imaging have reported a higher prevalence of CAD. In a study of 85 low-CAD-risk patients with ESLD, Kemmer et al reported a prevalence of prognostically significant coronary artery calcium score (CAC) of $>400$ to be present in $19(22 \%)$ with a prevalence of obstructive CAD on ICA to be $26 \% .^{6}$ In another study of 50 intermediate-to-high CAD-risk patients, severe obstructive CAD ( $\geq 70 \%$ luminal stenosis) was noted on coronary computed tomography angiography (CCTA) in $20 \% .^{7}$ Given the disparage data, and the scarce and extremely valuable commodity - the donor liver, routine testing for CAD in pre-LT patients is likely to continue. In addition, consensus is lacking regarding the methodology of testing for CAD in pre-LT patients. While AASLD practice guideline recommends dobutamine stress echocardiography (DSE) in all adult patients undergoing LT evaluation, ${ }^{2}$ a consensus statement from cardiovascular societies recommends a more measured approach, with testing only among those with multiple CAD-risk factors, although no preferred modality is specified. ${ }^{3} \mathrm{~A}$ pragmatic approach could be to rule out CAD by CCTA (with or without CAC) among low-risk patients and avoid repeat testing in these patients based on an established long warranty period in the setting of a negative test. ${ }^{8}$ Performance of CCTA may be limited by the presence of concomitant renal failure in some patients. A minority of these low-risk patients who may have anatomical CAD, or those with contraindications to CCTA or those with intermediate-to-high CAD risk may undergo functional testing, with the consideration of stress-first or stress-only imaging when MPI is being performed, especially with CZT systems. Repeat testing should be reserved for those with an initial abnormal test (to follow medical therapy or revascularization) or those who develop new symptoms while awaiting LT. Lastly, while DSE has been reported to be an effective screening test in pre-LT patients, many may not achieve target heart rate due to active beta-blocker therapy for portal hypertension. The high frequency of chronotropic incompetence noted in these patients likely results in a poor positive predictive value of DSE. ${ }^{9,10}$ Vasodilator stress MPI can be readily performed in these patients, as reported by Duvall et al, although it could be limited by resting hypotension. In addition, patients with ESLD 
have circulatory dysfunction characterized by arterial vasodilatation and the effectiveness of a vasodilator stress in them is uncertain. ${ }^{11,12}$ Among non-ESLD patients undergoing dipyridamole stress Rubidium-82 PET, a splenic switch-off phenomenon has been reported, wherein a visually reduced splenic tracer uptake has been identified to be a physiologic response to vasodilator stress. ${ }^{13}$ This finding has been complimented by a cardiac MRI-based perfusion study, that reported a lack of splenic switch-off with adenosine stress to be associated with a greater number of false-negative stress studies. ${ }^{14}$ These reports suggest that a failure of splenic switch-off could be an indicator of inadequate pharmacologic stress. While this phenomenon has not been specifically studied in ESLD patients, it may be of value to further evaluate splenic switch-off in ESLD patients to determine the adequacy of vasodilator stress among these patients who inherently have a physiological state of resting systemic vasodilatation.

\section{Disclosure}

Authors have nothing to disclose.

\section{References}

1. McAvoy NC, Kochar N, McKillop G, Newby DE, Hayes PC. Prevalence of coronary artery calcification in patients undergoing assessment for orthotopic liver transplantation. Liver Transpl. 2008;14:1725-31.

2. Martin P, DiMartini A, Feng S, Brown R Jr, Fallon M. Evaluation for liver transplantation in adults: 2013 Practice guideline by the American Association for the Study of Liver Diseases and the American Society of Transplantation. Hepatology. 2014;59:1144-65.

3. Lentine KL, Costa SP, Weir MR, Robb JF, Fleisher LA, Kasiske $\mathrm{BL}$, et al. Cardiac disease evaluation and management among kidney and liver transplantation candidates: a scientific statement from the American Heart Association and the American College of Cardiology Foundation. J Am Coll Cardiol. 2012;60:434-80.

4. Patel SS, Nabi E, Guzman L, Abbate A, Bhati C, Stravitz RT, et al. Coronary artery disease in decompensated patients undergoing liver transplantation evaluation. Liver Transpl. 2018;24:333-42.

5. Duvall L, Singhvi A, Tripathi N, Henzlova M. SPECT myocardial perfusion imaging in liver transplantation candidates. J Nucl Cardiol. 2018.

6. Kemmer N, Case J, Chandna S, Neff GW. The role of coronary calcium score in the risk assessment of liver transplant candidates. Transplant Proc. 2014;46:230-3.

7. Poulin MF, Chan EY, Doukky R. Coronary computed tomographic angiography in the evaluation of liver transplant candidates. Angiology. 2015;66:803-10.

8. Ostrom MP, Gopal A, Ahmadi N, Nasir K, Yang E, Kakadiaris I, et al. Mortality incidence and the severity of coronary atherosclerosis assessed by computed tomography angiography. $\mathbf{J}$ Am Coll Cardiol. 2008;52:1335-43.

9. Plotkin JS, Benitez RM, Kuo PC, Njoku MJ, Ridge LA, Lim JW, et al. Dobutamine stress echocardiography for preoperative cardiac risk stratification in patients undergoing orthotopic liver transplantation. Liver Transpl Surg. 1998;4:253-7.

10. Harinstein ME, Flaherty JD, Ansari AH, Robin J, Davidson CJ, Rossi JS, et al. Predictive value of dobutamine stress echocardiography for coronary artery disease detection in liver transplant candidates. Am J Transplant. 2008;8:1523-8.

11. Aydinalp A, Bal U, Atar I, Ertan C, Aktas A, Yildirir A, et al. Value of stress myocardial perfusion scanning in diagnosis of severe coronary artery disease in liver transplantation candidates. Transplant Proc. 2009;41:3757-60.

12. Davidson CJ, Gheorghiade M, Flaherty JD, Elliot MD, Reddy SP, Wang NC, et al. Predictive value of stress myocardial perfusion imaging in liver transplant candidates. Am J Cardiol. 2002;89:359-60.

13. Bami K, Tewari S, Guirguis F, Garrard L, Guo A, Hossain A, et al. Prognostic utility of splenic response ratio in dipyridamole PET myocardial perfusion imaging. J Nucl Cardiol. 2018;25:348-50.

14. Manisty C, Ripley DP, Herrey AS, Captur G, Wong TC, Petersen SE, et al. Splenic switch-off: A tool to assess stress adequacy in adenosine perfusion cardiac MR imaging. Radiology. 2015;276:732-40. 